\title{
EWOD-driven stirring in lab-on-a-chips: Dependence on the electrodes' geometry
}

\author{
R. Malk ${ }^{\mathrm{a}, \mathrm{b}}$, J. Theisen ${ }^{\mathrm{b}, \mathrm{c}}$, Y. Fouillet ${ }^{\mathrm{a}}$, L. Davoust ${ }^{\mathrm{c}, *}$ \\ ${ }^{a}$ CEA-LETI, 38041 Grenoble Cedex 9, France \\ ${ }^{\mathrm{b}}$ UMR LEGI, Grenoble Institute of Technology, 38041 Grenoble Cedex 9, France \\ ${ }^{\mathrm{c}}$ Electromagnetic Processing of Materials (EPM) Group, Materials and Processes Science and Engineering Laboratory (SIMAP), Grenoble Institute of Technology \\ (GRENOBLE-INP), 38402 Saint Martin d'Hères, France
}

\begin{abstract}
Electrowetting on dielectrics (EWOD) with coplanar electrodes is increasingly used in microsystems. This paper enlightens the physical mechanisms of stirring flows in sessile droplets actuated by oscillating EWOD with potential applications to biochemical reaction enhancement or cells handling. Depending on electrodes design (circular vs. square electrodes), EWOD-induced convective flow can be observed to switch from toroidal to quadripolar flows. Due to the coplanar geometry of the electrodes, a model can be proposed which helps to interpret EWOD-induced flow as a result of capillary streaming. Numerical calculations are compared with imaging of drops and demonstrate clearly the essential role of oscillating EWOD as a flow actuator.
\end{abstract}

\section{Introduction}

Electrowetting on dielectric EWOD) is currently used as a driving mechanism in microsystems like variable focal lenses [1], displays [2] or digital lab-on-a-chip devices for clinical diagnostics [3]. Used in AC mode at a frequency, $f \sim 1 \mathrm{kHz}$ or more, EWOD is currently identified as a means to manipulate drops along a surface [4]. But EWOD can also be used at a lower frequency in a regime said oscillating EWOD $(f \sim 100 \mathrm{~Hz})$. This permits to induce shape oscillations of a sessile drop which in return generates a Stokes drift along its liquid surface. Oscillating EWOD has recently been proposed as a mixing promoter $[5,6]$ in the popular needle configuration of electrowetting. ${ }^{1}$

In this paper, it is demonstrated how EWOD-induced drop stirring can also be provided with a coplanar electrode configuration compatible with the purpose of microsystem integration since there is no need for any external needle. As a typical application, present authors recently demonstrated how EWOD-induced drop flow can be monitored to focus colloids [7] or U373B cells [8]. From an original modeling based on wave streaming, supported by drop experiments with air as ambient phase, the present paper gives new insight into EWOD-induced drop stirring.

\section{Materials and methods}

Technological steps are performed from a $200 \mathrm{~mm}$ silicon wafer and EWOD chips are composed of three main layers: the electrodes

\footnotetext{
* Corresponding author.

E-mail address: laurent.davoust@simap.grenoble-inp.fr (L. Davoust).

1 A sessile drop is staying over a dielectric film with a first electrode underneath and a needle electrode above which is put in capillary contact with drop apex.
}

(200 nm thick AlCu); the dielectric layer (600 nm thick Si3N4); and, finally, the hydrophobic layer ( $1 \mu \mathrm{m}$ thick SiOC). These materials enable to integrate the whole fabrication process into cleanrooms with no compromise on EWOD performance and a good reproducibility during assays. More details of the process flow are described in Fig. (1) as well as in [7].

The simple chip design used here is made from two half disks shaped coplanar electrodes (radius $=2 \mathrm{~mm}$ ) separated by a $3 \mu \mathrm{m}$ gap (Fig. 2). The prior advantage of this coplanar electrode configuration - compared with the classical needle electrode configuration - is that droplet oscillations are not disturbed by any needle counter-electrode. Consequently, image analysis is therefore facilitated and theoretical modeling is made possible. A $1.5 \mu \mathrm{l}$ droplet of phosphate buffered saline (PBS) is placed on the chip surface with a micropipette. AC electrical signal is generated from a generator (Yokogawa FG120) and a home-made amplifier. A CCD camera (Pixelfly $200 \mathrm{XS}$ ) and a zoom lens are used for image acquisition. The camera is placed so as to visualize droplet profiles (Fig. 3). A LED is used as a stroboscopic lighting source placed behind the droplet in order to create a backlight and to minimize light reflection. The LED is plugged to the same generator. The frequencies of $\mathrm{AC}$ actuation and stroboscopic lighting are equal, and by shifting the phase lag between both signals the instantaneous shape of the droplet can be clearly imaged. Time-dependent droplet profiles are acquired and analyzed using home-made software.

Drop convective flow is simultaneously analyzed by imaging fluorescent beads (mean diameter: $10 \mu \mathrm{m}$ ) and using a software dedicated to micro-PIV (Particle Image Velocimetry). The laser sheet used to induce beads fluorescence is provided by a laser source (Melles Griot: $5 \mathrm{~mW}$ ) and two cylindrical lenses. 
(a)

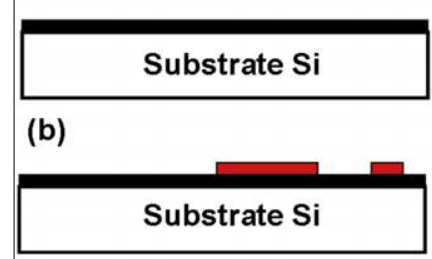

(d)

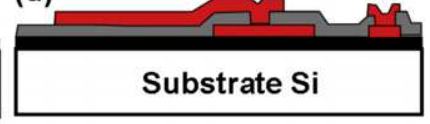

(e)

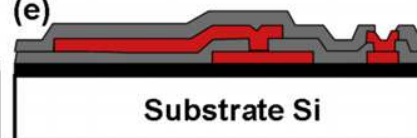

(c)
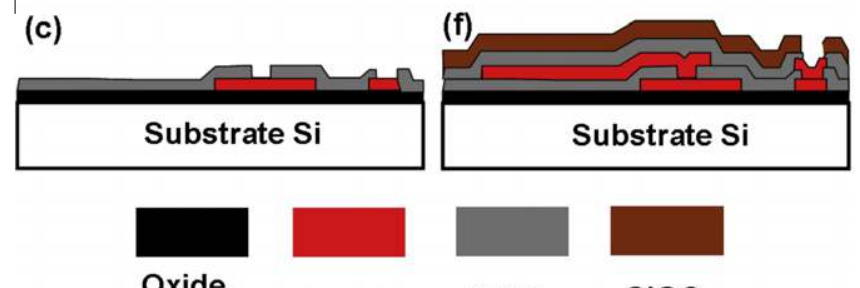

silicon

Metal

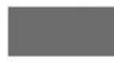

Si3N4

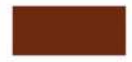

SiOC
Fig. 1. (a-f) Process flow.

\section{Impact of the electrodes geometry on drop flows and supporting theory}

Experimentally, two different chip designs are investigated: a first geometry with two half-circular electrodes (see Figs. 2(a) and 4) and a second geometry with two squared electrodes (see Figs. 2(b) and 6), which is usually involved in EWOD-based labon-a-chips (LOC).

\subsection{Isotropic electrowetting}

When coplanar electrodes exhibit a circular geometry in favor with isotropic electrowetting of a drop, stirring is found to remain perfectly 2-D axisymmetric with the arising of a discrete number of toroidal vortices driven by the resonant eigenmode or, equivalently, the frequency of the AC actuation. In Fig. 4, top view (a) and side view (b) of the flow are made evident for an input frequency, $f=110 \mathrm{~Hz}$, with the arising of two permanent toroidal structures (oscillating mode $k=2$ ). This flow is steady and the velocity is estimated to be $\sim 1 \mathrm{~mm} / \mathrm{s}$.

\subsubsection{Analysis of vortex position vs. frequency}

The vortices located within a meridian plane of the droplet are steady and, interestingly, when input frequency is changed, they move to a new position along the droplet surface [7]. Increasing the actuation frequency, the vortices get closer to the wetting plane and their mean vorticity decreases. Our experiments demonstrate a surprising reproducibility and reversibility of the vortex locations when actuation frequency ranges from $3 \mathrm{kHz}$ to $150 \mathrm{~Hz}$ and vice versa. The droplet oscillations induced by AC EWOD are also simultaneously observed by shifting laser lighting to stroboscopic lighting. The change in input frequency also leads to a change in oscillation mode. At $110 \mathrm{~Hz}$, the amplitude of drop shape oscillations is found maximum at the apex and at mid-height of the droplet, which corresponds to the second radial eigenmode. Clearly, it can be demonstrated that drop stirring flow is induced by capillary standing waves, which are excited at the drop surface by oscillating EWOD.

\subsubsection{EWOD-induced streaming current as a surface driver}

For a frequency $f=\frac{\omega_{k}}{2 \pi}$ of the AC voltage, the drop oscillates with a frequency $f_{g}=\frac{\xi_{k}}{2 \pi}=\frac{\omega_{k}}{\pi}$ since the electric force on the contact line scales as the square of the applied voltage. The shape of the oscillating drop can be fairly described as a half-sphere modulated by a radial deformation, $r=R_{0}+A_{k} \sin \left(\xi_{k} t+\psi_{k}\right) P_{k}(\cos (\theta))$, where $A_{k}$ is the amplitude of drop oscillations for the wave mode $k, \psi_{k}$ is the associated phase lag, $P_{k}$ is $k$ th-degree Legendre polynomial and $R_{0}$ is the mean radius calculated from a sessile drop supposed to be a half-sphere when the voltage frequency is large enough $(>1 \mathrm{KHz})$. Hence, it is possible to calculate the normal component of the surface velocity, $U_{k}^{\infty}=\frac{A_{k} \xi_{k}}{k} \frac{d P_{k}(\cos \theta)}{d \theta} \cos \left(\xi_{k} t+\psi_{k}\right)$, that is associated with standing capillary waves. Considering the theory of steady streaming [9], it can be demonstrated that a steady velocity, $U_{k}^{s}$, referred to as streaming current, is provided at the boundary of a Stokes layer staying along the inner of the mobile drop surface:

$U_{k}^{s}=-\frac{3}{8 R_{0} \xi_{k}} \frac{d}{d \theta}\left[\left(U_{k}^{\infty}\right)^{2}\right]$

with

$U_{k}^{\infty}=\frac{A_{k} \xi_{k}}{k} \frac{d P_{k}(\cos \theta)}{d \theta}$. (a)

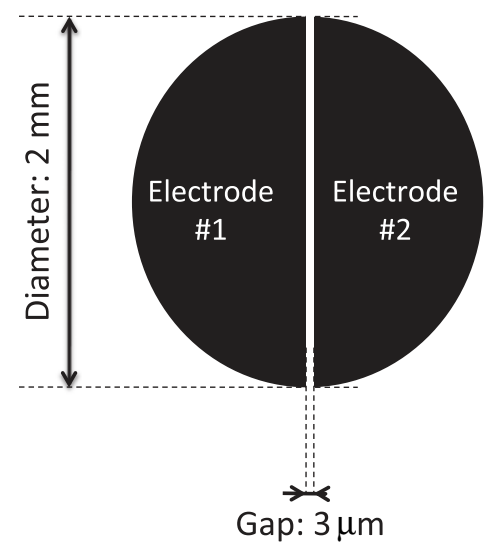

(b)

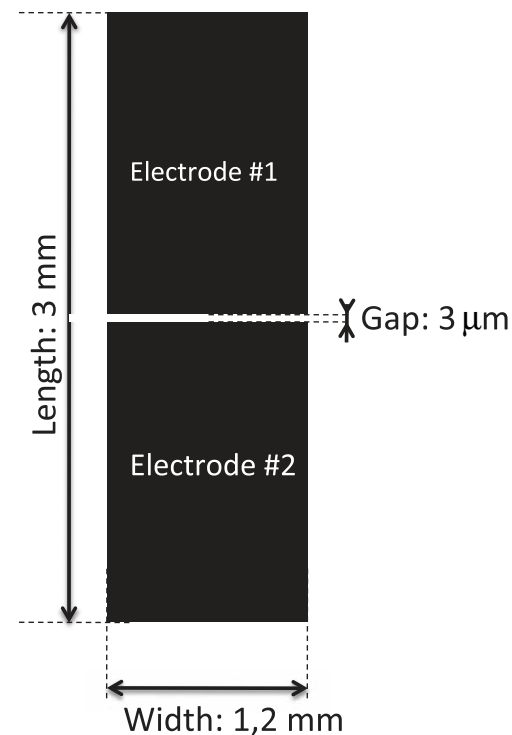

Fig. 2. Design of the electrode pairs: (a) two half-circular electrodes; (b) two squared electrodes. 


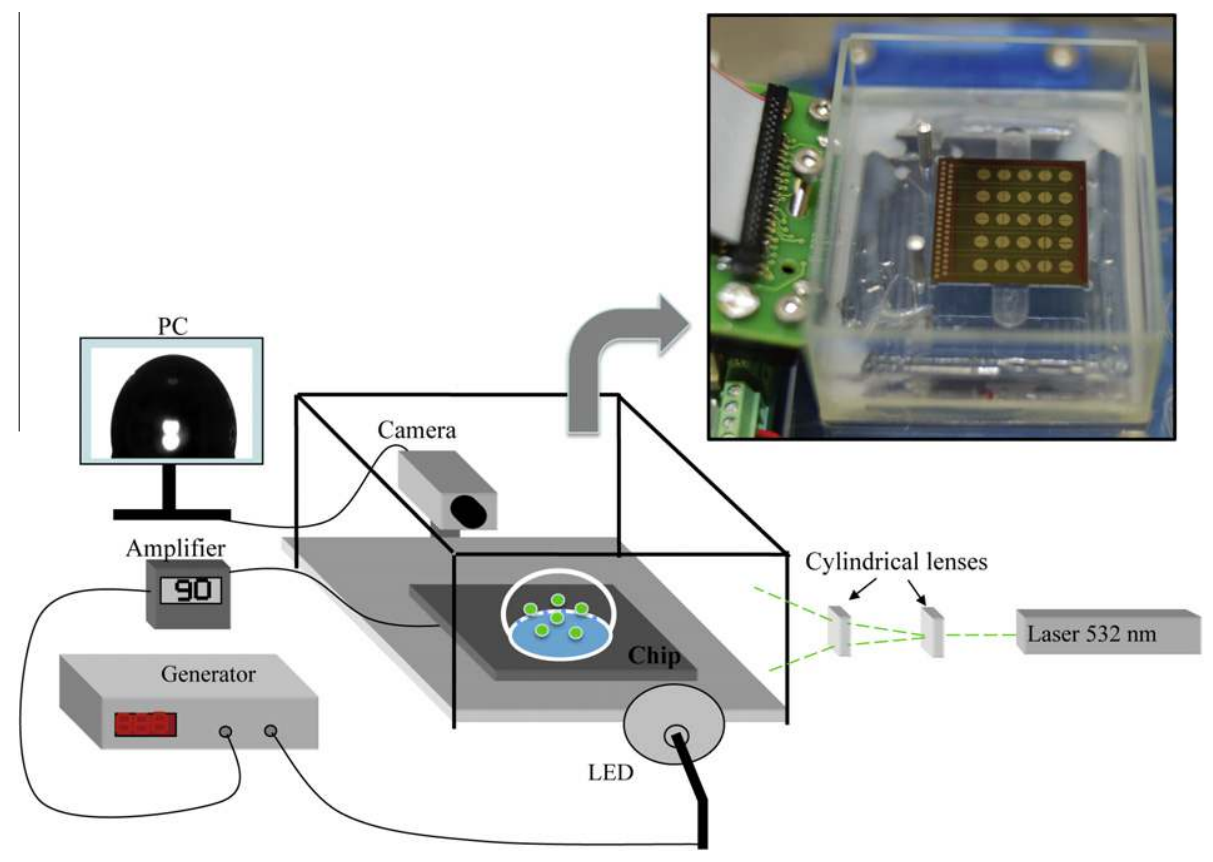

Fig. 3. Experimental setup and photograph of chip array.

(a) Top view

(b) Side view

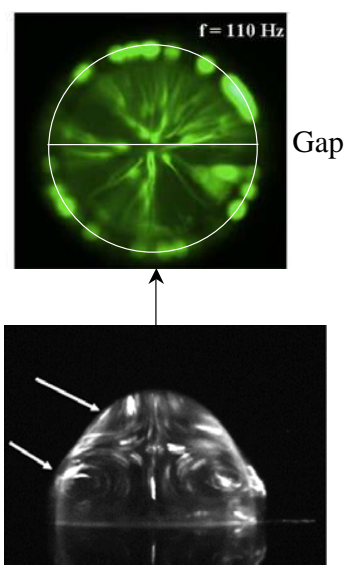

Width or length (c) Simulation

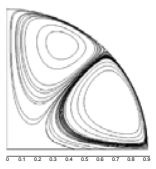

Fig. 4. An axisymmetric flow within a sessile drop under oscillating EWOD (chip with two half-circular electrodes, actuation frequency: $f=110 \mathrm{~Hz}$, wave number: $k=2$ ) as observed from (a) above, (b) in drop cross-section. Arrows indicate the presence of two toroidal vortices. For sake of comparison, (c) toroidal vortices as calculated in a meridian plane.

In our case, the thickness of the Stokes layer is much thinner than drop radius, $\delta_{s}=\mathcal{O}\left(\sqrt{\frac{\eta}{\rho \xi_{k}}}\right) \ll R_{o}$, with $\eta$ and $\rho$, the viscosity and the density of the drop, respectively. And consequently, the streaming velocity can be considered as a surface boundary condition for a convective flow, which may be calculated inside a permanent half spherical drop (time-averaged shape).

\subsubsection{Numerical calculations vs. experiments}

From the literature [10], the streaming Reynolds number, $R_{k=2,4 \ldots}^{s}=\frac{\rho\left(U_{k=2,4 \ldots}^{\infty}\right)^{2}}{\xi_{k} \eta}$, depends on the oscillating mode. In our case, its value is never negligibly small $\left(R_{k}^{s} \sim 10-100\right)$ and as a consequence, the drop flow cannot be considered as creeping. Hence,

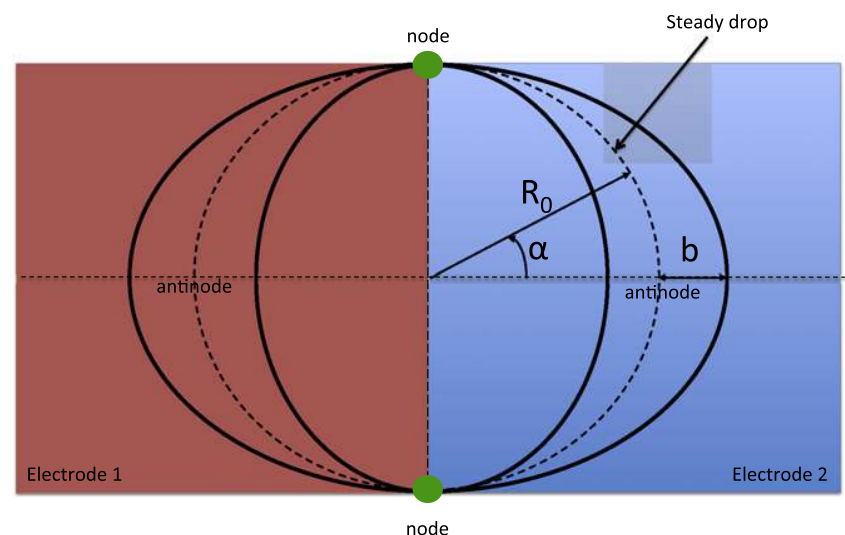

Fig. 5. 2-D streaming model as an attempt to explain quadripolar flows observed in a sessile drop on a pair of squared electrodes.

numerical simulations are conducted under $\mathrm{Comsol}^{\circledR}$ software (Finite Element Methods) in order to predict the drop flow induced by EWOD-streaming and to compare it with our velocimetry experiments performed from tracking of fluorescent beads. The drop considered for present calculations is a half-sphere ${ }^{2}$ for which all the oscillating modes are time-averaged. The expression (1) is therefore introduced as a boundary condition along the drop surface for each mode considered. Due to 2-D axisymmetry, flow calculation is performed within a meridian cross-section: two slip conditions are imposed along the vertical drop axis and the underlying substrate.

Top and side views of the inner flow as produced under oscillating EWOD $(f=110 \mathrm{~Hz}$, mode $k=2)$ are displayed in Fig. 4(a) and (b). Two (axisymmetric) toroidal vortices are consistently made evident from imaging of fluorescent beads and for sake of comparison, streamlines are calculated within a meridian cross-section (Fig. 4(c)). A fair agreement is therefore

\footnotetext{
${ }^{2}$ This assumption is not so restrictive since quite often, EWOD delivers a contact angle close to $\pi / 2$.
} 


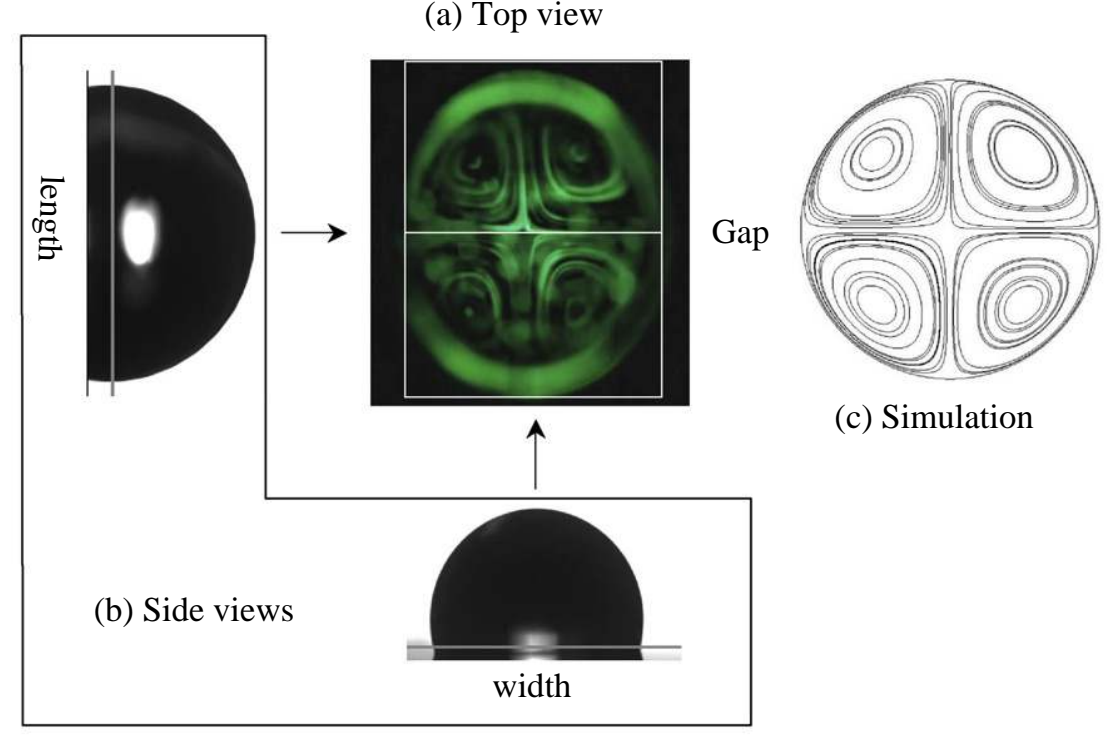

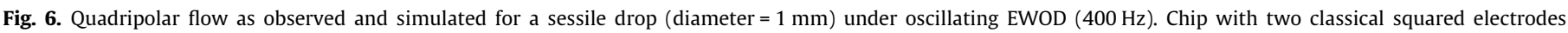
$($ size $=800 \mu \mathrm{m})$.

demonstrated and it is worthy to mention that the velocity measured at the vicinity of the drop apex is of the same order as the numerically calculated velocity whatever the relative mode amplitude is: $u \simeq 2 \mathrm{~mm} / \mathrm{s}$ for $\frac{A_{k=2}}{R_{0}}=0.045$ and $u \simeq 6.5 \mathrm{~mm} / \mathrm{s}$ for $\frac{A_{k=2}}{R_{0}}=0.06$.

A fair agreement between experiments and numerical predictions is also found for oscillation modes $k=4$ and 6 , for which the actuation frequency, $f$, is never larger than $450 \mathrm{~Hz}$. Beyond this critical frequency, it is not possible to observe the toroidal flow expected for mode $k=8$ : the drop flow is no more axisymmetric and steady. When imaging streamlines from the top, a bipolar vortex with rotation about an arbitrary axis is found.

\subsection{Anisotropic electrowetting: local pinning of the contact line}

Now, one considers EWOD of a drop one millimeter in diameter which is straddling two actuated squared electrodes. ${ }^{3}$ Considering a typical size of the electrodes $(\sim 1 \mathrm{~mm})$, typically smaller than the size of the wetting area, the drop is free to wet the electrodes pair along the length. But along the gap, the contact line can extend up to the electrodes edge. In that case, the contact line is locally trapped due to a wetting singularity while it is still free to move along the length of the electrodes pair. This break-up of wetting symmetry causes a fundamental change in stirring flow:

- for an actuation frequency no larger than $150 \mathrm{~Hz}$, the drop flow remains axisymmetric and quite similar to the one previously observed for mode $k=2$,

- for an actuation frequency ranging between 150 and $300 \mathrm{~Hz}$, the drop flow is no more axisymmetric and becomes strongly 3-D: it exhibits two recirculating flows, as viewed along the electrodes gap, and four recirculating flows as viewed along the length of the electrodes,

\footnotetext{
3 The squared electrode is the usual geometry involved in EWOD-based LOCs.
}

- for an actuation frequency larger than $300 \mathrm{~Hz}$ and beyond (up to $600 \mathrm{~Hz}$ at least), a surprisingly reproducible quadripolar flow can be imaged above the drop, which exhibits four steady vortices (Fig. 6).

As an attempt to interpret this flow configuration, we consider successive stretching-contraction cycles of the drop along the electrode pair, as depicted in Fig. 5. Standing waves are again considered with the presence of two nodes at the locations where the contact line is trapped, and the presence of two anti-nodes at the locations where the oscillating motion of the contact line is the largest. Here again, a streaming model makes sense but this time, the drop geometry is approximated as a flat cylinder (Fig. 5) with a base radius, $r=R_{0}+b \sin (\xi t) \cos (\alpha)$, where $b$ is the amplitude of antinodes motion, $R_{0}$ is the time-averaged base radius, $\xi$ is the oscillation pulsation and $\alpha$ is referred to as the polar angle defined in the wetting plane. From these considerations, the streaming velocity,

$U^{s}=-\frac{3 b^{2} \xi}{8 R_{0}} \sin (2 \alpha)$

is considered as a boundary condition to inject as a slip velocity along the contact line. It is interesting to notice that, despite the simplicity of this model, the quadripolar flow is recovered, as demonstrated in Fig. 6, and the calculated velocity scale is found to be of same order as the experimental one: $u \simeq 1 \mathrm{~mm} / \mathrm{s}$.

\section{Concluding remarks}

Numerical simulations of EWOD streaming-induced drop flow are favorably compared with experimental results. From this, it is demonstrated how the shape of the electrodes (circular, squared...), the frequency of the applied voltage, the aspect ratio of the drop size to the electrode size and even the aspect ratio of the drop size to the electrode gap, allow the end-user to monitor the flow patterning within a drop under EWOD. From this, design guidelines on EWOD chips and frequency monitoring can be 
formulated. Specific applications can be addressed such as flow-focusing of biological material [7], functionalization of solid spots [8], supernatants handling, mixing [4], etc.

\section{References}

[1] B. Berge, J. Peseux, Eur. Phys. J. E 3 (2000) 159-163.

[2] R. Hayes, B. Feenstra, Nature 425 (2003) 383-385.
[3] M.G. Pollack, V.K. Pamula, V. Srinivasan, A.E. Eckhardt, Expert Rev. Mol. Diag. 11 (2011) 393-407.

[4] R. Malk, A. Rival, Y. Fouillet, L. Davoust, ASME Conf. Proc. 54501 (2010) 239-248.

[5] R. Miraghaie, J. Sterling, A. Nadim, NSTI-Nanotech 2 (2006) 610-613.

[6] F. Mugele, A. Staicu, R. Bakker, D. van denEnde, Lab. Chip 11 (2011) 2011-2016.

[7] R. Malk, Y. Fouillet, L. Davoust, Sensor Actuat. B 154 (2011) 191-198.

[8] R. Malk, Y. Fouillet, L. Davoust, Microelectron. Eng. 88 (2011) 1745-1748.

[9] G. Batchelor, Introduction to Fluid Mechanics, Cambridge University Press, 2000

[10] J. Stuart, J. Fluid Mech. 87 (1966) 624-673. 